\title{
NOTES SUR UNE INSCRIPTION DE LA BAIE DE CADIX: L'ÉPITAPHE D'OPTATA ERENNIA
}

\section{NOTES ON AN INSCRIPTION OF THE BAY OF CÁDIZ: THE EPITAPH OF OPTATA ERENNIA}

\author{
ANTHONY ÁLVAREZ MELERO* \\ MANUEL J. PARODI ÁLVAREZ**
}

\begin{abstract}
Résumé: L'inscription funéraire du Optata Erennia est toujours l'un des rares exemples d'épigraphes romaines apparu dans une région par ailleurs si riche en sites archéologiques, comme la ancienne ville de Puerto Real (Cadix). Nous aborderons dans les lignes suivantes son étude et identification, les caractéristiques et la nature du cette bref texte funéraire.
\end{abstract}

Mots-clés: Optata Erennia, Puerto Real, Cadix, épigraphe, funéraire, affranchis, nom, surnom.

L'inscription funéraire d'Optata Erennia est l'un des rares exemples d'épigraphes romaines apparues dans une région par ailleurs très riche en sites archéologiques, comme l'ancienne ville de Puerto Real (Cadix). Nous aborderons dans les lignes suivantes son étude et identification, les caractéristiques et la nature de ce bref texte funéraire ${ }^{1}$.

* Collaborateur scientifique ULB - Universidad de Sevilla.

** Universidad de Sevilla. Grupo PAI HUM 402

1. Cet article a été rédigé dans le cadre du projet « Generación de elites y vertebración provincial: la Bética romana » (HAR200804820-C04-01), du VI Plan national de recherche scientifique, développement et innovation technologique du ministère espagnol de la Science et de l'Innovation, cofinancé avec des fonds européens FEDER. Nous tenons également à remercier Mmes Marie-Thérèse Raepsaet-Charlier (ULB) et Monique Dondin-Payre (CNRS) ainsi que Mme Helena Gimeno Pascual du centre CIL II $^{2}$ de l'Université d'Alcalá de Henares sans oublier M. José Luis Gamallo et toute l'équipe du centre Hispania Epigraphica de l'Université complutense de Madrid pour leurs conseils et avis. Les fautes qui subsisteraient

\begin{abstract}
The funerary inscription of Optata Erennia is one among the very short list of examples of Latin epigraphs appeared up to the date in an area so deeply rich in Roman sites as the territory belonging to the old town of Puerto Real (near Cadiz). In the following pages we shall get in touch with this epigraph, studying its meaning, nature and the oddities of its historical appearances.
\end{abstract}

Key words: Optata Erennia, Puerto Real, Cadiz, epigraph, funerary, freedmen, name, last name.

En 1962, l'archéologue espagnole Mª . J. Jiménez Cisneros publiait un ensemble de 35 épitaphes provenant de fouilles menées dans une nécropole de Cadix. Parmi celles-ci, rangée sous le numéro 16, figurait l'inscription d'Optata Erennia ${ }^{2}$. Une vingtaine d'années plus tard, J. González Fernández ne put la retrouver pour son édition des inscriptions de la province de Ca$\mathrm{dix}^{3}$ et l'épigraphiste sévillan dut se résoudre à suivre les indications de son prédécesseur. Pour ces mêmes motifs, notre épigraphe est complètement passée sous silence dans les éditions d'inscriptions du Musée archéologique de Cadix publiées par Ma . D. López de la Orden, avec A. Ruiz Castellanos ${ }^{4}$ d'abord, puis seule ${ }^{5}$.

nous sont en revanche entièrement imputables. Signalons aussi qu'une version abrégée de cet question paraitra prochainement dans Latomus.

2. Ma.J. Jiménez (1960: 295-ss.).

3. J. González (1982: fig. 424).

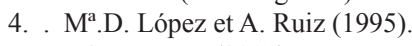

5. . Mª .D. López (2001). 


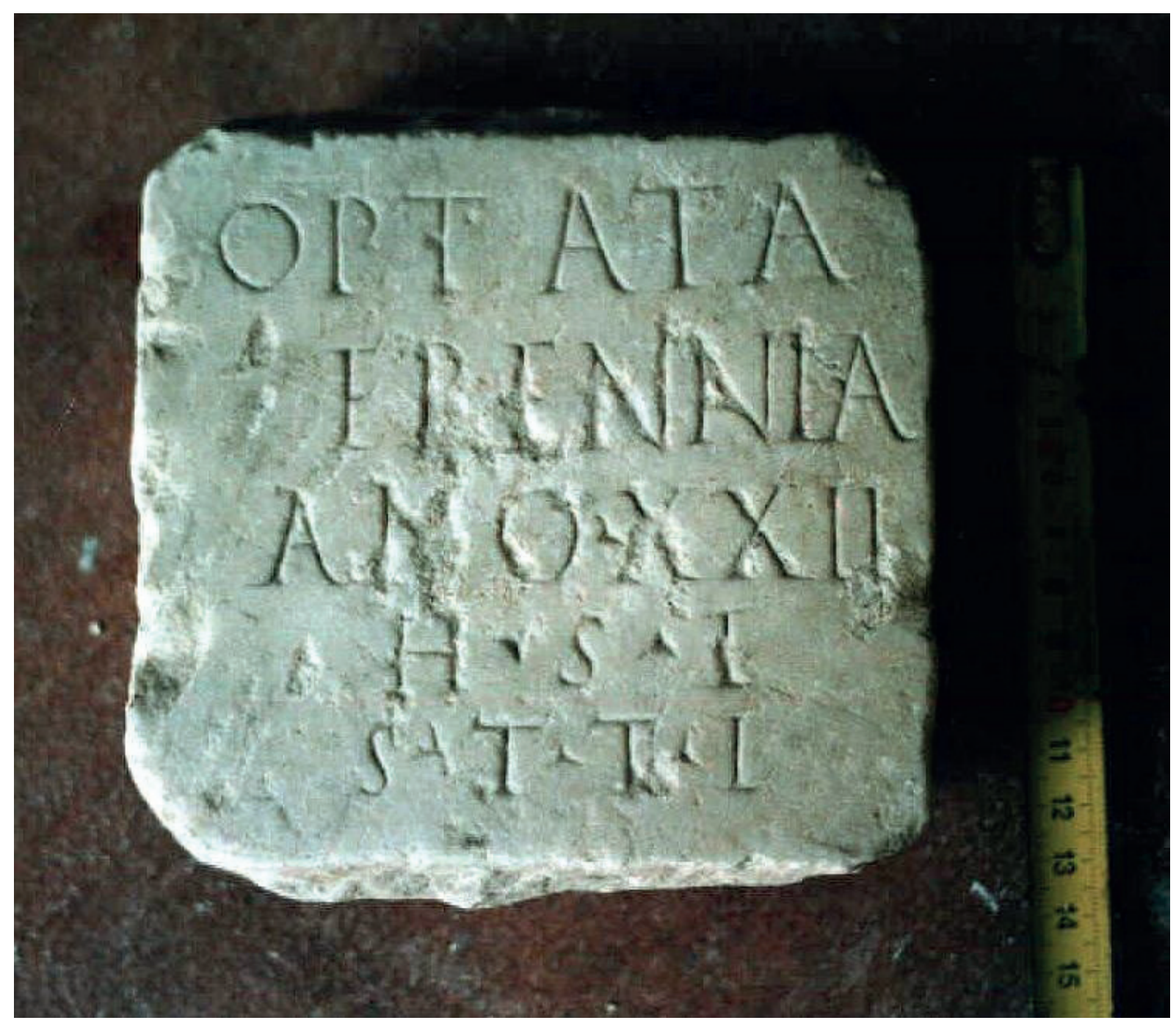

Fig. Inscription d'Optata Erennia

Toutefois, au début des années 2000, cette inscription a reparu dans la ville de Puerto Real, à une dizaine de kilomètres à l'Est de l'antique cité phénicienne, avant de disparaître une nouvelle fois. La pierre, découverte en remploi ${ }^{6}$, faisait partie du mur d'un édifice situé Calle La Palma, en plein centre historique de l'ancienne cité portuaire (fondée par le royaume de Castille à la fin du $\mathrm{XV}^{\mathrm{e}}$ siècle).

Ce fait peut susciter bien des interrogations puisqu'il nous empêche d'élucider la question de la provenance

6. Il est habituel que les plaques de ce genre se soient trouvées encastrées dans un mur bâti une pierre locale, dite " ostionera » faite d'un mélange de silice et de carcasse d'huîtres («ostión») et caractéristique de la zone, lors de sa construction à une époque incertaine. exacte de cette pierre, entre Cadix et Puerto Real. Doiton penser que la chercheuse de Cadix s'était mal exprimée ? Faut-il au contraire considérer que l'épitaphe d'Optata Erennia a disparu aussitôt après sa découverte pour ensuite se retrouver encastrée dans le mur d'une habitation de Puerto Real ${ }^{7}$ ? Le mystère demeure entier et il nous semble à l'heure actuelle difficile de faire la lumière sur cette question puisque il ne nous a pas été possible d'accéder aux archives de $\mathrm{M}^{\mathrm{a}}$. J. Jiménez Cisneros, aujourd'hui décédée.

7. Cette hypothèse semble plausible à en croire $\mathrm{M}^{\mathrm{a}}$. J. Jiménez Cisneros qui affirmait (1960: 296) que certaines inscriptions lui furent présentées par des ouvriers qui ont travaillé sur les chantiers. 
Quoi qu'il en soit, il nous a été permis de prendre un cliché qui confirme à tous points de vue la lecture de son premier inventeur.

Il s'agit donc d'une plaque funéraire de pierre locale, de $13 \mathrm{~cm}$ de haut, $13,5 \mathrm{~cm}$ de large et $3 \mathrm{~cm}$ d'épaisseur. Ce texte ne présente pas de ligatures et ses signes de ponctuation sont triangulaires (lignes 3 à 5).

Les lettres de la première et de la deuxième lignes ont une hauteur de $1,8 \mathrm{~cm}$; celles de la troisième 1,5 $\mathrm{cm}$; celles de la quatrième $1,3 \mathrm{~cm}$ et celles de la dernière $1,2 \mathrm{~cm}$.

Les espaces interlinéaires sont de $0,8 \mathrm{~cm}$ entre la première et la deuxième lignes et de $0,6 \mathrm{~cm}$ entre les autres.

Le texte est le suivant :

\section{OPTATA}

\section{ERENNIA}

\section{ANO $\beta$ XXII}

\section{$\mathrm{H} \beta \mathrm{S} \beta \mathrm{E}$}

\section{S B T B T B L}

Optata / Erennia, / an $<\mathrm{n}>$ o(rum) XXII, / h(ic) s(ita) e(st). ${ }^{5} \mathrm{~S}$ (it) t(erra) t(ibi) 1(euis).

« Ci-gît Optata Erennia, âgée de vingt-deux ans. Que la terre te soit légère $»$.

Si la mise en page de l'inscription est claire au point que l'on devine même l'axe vertical autour duquel le texte est disposé (on le voit même assez clairement ligne 1), il faut toutefois mettre l'accent sur quelques petites variantes de langage. En premier lieu, il convient de souligner l'absence de « $\mathrm{H}$ » initial pour transcrire le gentilice Herennius. Ensuite, la graphie ANO qui renvoie plutôt au vocable an $<\mathrm{n}>\mathrm{o}$ (rum), tournure au génitif assez habituelle à Cadix et dans ses environs.

Notons aussi l'inversion du gentilice et du surnom, documentée à maintes reprises dans la Péninsule ${ }^{8}$ comme le résume ce tableau ${ }^{9}$ :

8. Deux cas sont douteux : CIL, II, 2945 (= AE, 1994, 1027) (Salvatierra) ainsi que $A E, 1994,859=H E p, 6,102$ (Augusta Emerita) qui mentionnent respectivement Quinta Fabia et Fausta Coelia, deux femmes qui pourraient porter un prénom, habitude rare mais non inusitée et parfois difficile à apprécier : cf. M. Kajava (1994).

9. De notre développement.

\begin{tabular}{|c|c|}
\hline \multicolumn{2}{|c|}{ Hispania Vlterior Baetica } \\
\hline Galla Servilia ${ }^{10}$ & Italica (Santiponce) \\
\hline Nigrina Se[- - -]tia ${ }^{11}$ & $\begin{array}{c}\text { Iulipa } \\
\text { (Zalamea de la Serena) }\end{array}$ \\
\hline \multicolumn{2}{|c|}{ Hispania Vlterior Lusitania } \\
\hline Alucius Roscius C. $1 .^{12}$ & Villamesías \\
\hline Fecunda Herennia ${ }^{13}$ & Metellinum (Medellín) \\
\hline Severa Mania L. f. ${ }^{14}$ & $\begin{array}{c}\text { Caesarobriga } \\
\text { (Talavera la Reina - Oropesa) }\end{array}$ \\
\hline \multicolumn{2}{|c|}{ Hispania Citerior Tarraconensis } \\
\hline Aius Antonius ${ }^{15}$ & Clunia \\
\hline Felicia Iunia ${ }^{16}$ & Valeria (Valera de Arriba) \\
\hline
\end{tabular}

10. CIL, II, $1149=$ ERIT, $116=$ CILA, Se, 2, $449:$ Galla Blast $/$ Seruilia Su/perati. Cette plaque carrée de marbre aujourd'hui conservée dans la collection de la cathédrale de Tolède fait référence à une Servilia Galla, fille de (Servilius) Blastus et épouse de Superatus, éventuellement identifiable à Fabius Superatus décédé à 80 ans $(C I L$, II, $1146=C I L A, \mathrm{Se}, 2,440)$. J. González Fernández, dans son édition des CILA, p. 103, fait de Galla l'affranchie d'un Blast(i?)us Servilianus Superatus : Galla Blasti / Seruilia(ni) Su/perati [lib(erta)]. Cette lecture est rejetée par A. Canto (cf. HEp, 4, 733, p. 261) au motif qu'il y a assez d'espace sur la pierre pour les compléments proposés par J. González Fernández. De plus, si l'on suit l'interprétation de l'épigraphiste sévillan, l'anthroponyme «Blasti » ne cesse pas de poser problème : s'agit-il du gentilice Blastius, un hapax à l'heure actuelle, ou bien faut-il plutôt y déchiffrer le cognomen Blastus ? Dans ce dernier cas, le patron de Galla porterait trois surnoms, ce qui nous paraît difficile à accepter. Notre lecture, en revanche, permet donc de contourner ces difficultés, sans nécessiter d'adjonctions contraires à l'aspect et à la disposition du texte sur la pierre.

11. CIL, II, $2356=C I L, \mathrm{II}^{2} / 7,915:$ Maura Q(uinti) Valer $[i] /$ serua ann(orum) XXI[I?] / h(ic) s(ita) e(st) s(it) t(ibi) t(erra) l(euis). I Q(uintus) Valerius \& Mode(- - ) ann(orum) LXXV / h(ic) s(itus) e(st) s(it) t(ibi) t(erra) l(euis). / Nigrina Se+[- - -]/tia ann(orum) L / h(ic) s(ita) e(st) s(it) t(ibi) t(erra) l(euis).

12. JIWE, I, 188 : Alucius / Roscius C(ai) l(ibertus) / h(ic) s(itus) e(st) / Iud(a)eus / ACOLI C S. La lecture de cette inscription est controversée mais il est hautement probable qu'il y a une inversion entre le gentilice de cet affranchi, Roscius, et son surnom, Alucius, qui renvoie à l'anthroponyme indigène $\mathrm{Al}(1) \mathrm{u}(\mathrm{c}) \mathrm{q}(\mathrm{u})$ ius attesté à maintes reprises essentiellement en Lusitanie : voir J.M. Abascal (1994: 268); id., M. Navarro et al. (2003: 82 y 373); J.M. Vallejo (2005: 125-127). Autre lecture chez D. Noy, JIWE I, p. 261-262.

13. CIL, II, 614 : Fecunda Here/nia h(ic) s(ita) e(st) p(edum) XII / 'e 'x p (ondo) arg(enti) Famelius / c(urauit) d(edicandum).

14. CIL, II, 945:D(is) M(anibus) / Seuer/a Ma/nia L(uci) f(ilia) /5 ann(orum) X[---] / h(ic) s(ita) e(st) s(it) t(ibi) t(erra) l(euis). I Seuerin/a Seueri / f(ilia) mat(er) ${ }^{10}$ f(aciendum) cur(auit).

15. CIL, II, $2786=$ ERClunia, 36 : Attuae Bou/tiae Bouti f(iliae) / Intercatiensi / an(norum) XXXII /s Aius Antonius / uxo(ri) s(uae) f(aciendum) c(urauit)..

16. CIL, II, 3195 : Felicia Iunia / an(norum) XXIII h(ic) s(ita) / est s(it) t(ibi) t(erra) l(euis). 


\begin{tabular}{|c|c|}
\hline \multicolumn{2}{|c|}{ Hispania Citerior Tarraconensis } \\
\hline Fuscus Bitius $^{17}$ & Calagurris (Calahorra) \\
\hline Marcelus Aurelius $^{18}$ & Lara de los Infantes \\
\hline Rustica Baebia $^{19}$ & Saguntum (Sagonte) \\
\hline
\end{tabular}

L'inversion entre le gentilice et le surnom a fait l'objet d'une fine analyse de la part d'I. Kajanto dans le cadre d'une étude sur l'onomastique féminine. Le savant finlandais, sur la base d'un échantillon tiré du $C I L$, VI, explique qu'il s'agit d'un reliquat de la nomenclature servile, caractéristique des débuts de l'Empire ${ }^{20}$, mais des variations locales existent.

En effet, il faut souligner l'existence de pratiques régionales, comme en Cisalpine (surtout dans les régions IX et XI), où c'est sous la forme d'un prénom qu'il faut parfois interpréter l'anthroponyme qui précède le gentilice des citoyens romains d'origine indigène à la fin de l'époque républicaine et au début de l'Empire ${ }^{21}$.

En ce qui concerne les provinces hispaniques, on constate que la majorité des attestations se réfère à des femmes mais on ne peut rien affirmer ni sur leur statut juridique ni sur la chronologie. Pour cette raison, rien ne permet de certifier qu'Optata Erennia ait été une affranchie. Il est vrai que l'examen des inscriptions publiées par $M^{\mathrm{a}}$. J. Jiménez Cisneros nous dévoile l'existence de personnages pour lesquels formuler l'hypothèse d'une telle extraction ne parait pas farfelu ${ }^{22}$. Un doute raisonnable peut donc se faire jour. Toutefois, la concision du formulaire de l'épitaphe incite à une prudence que renforce également le contexte archéologique. En effet, il semble bien que les épigraphes présentées par l'archéologue espagnole aient été collectées hors de leur emplacement originel, une nécropole, et cette dernière, pour autant que nous sachions, n'était pas exclusivement réservée à des affranchis. Cela explique qu'on y ait découvert des épitaphes appartenant à des personnalités relevant de différentes

17. CIL, II, 2984 (p. 937) $(=I L S, 2516)=$ ERRioja, 7 : Iulius Lon/ginus Doles / Biticenti f(ilius) Bes/sus eques alae ₹F Tau(rianae) Tor(quatae) Vic(tricis) c(iuium) R(omanorum) / an(norum) XL aer(orum) XXII h(ic) s(itus) e(st) / Sulpicius Susulla / et Fuscus Bitius / h(eredes) ex t(estamento) f(aciendum) c(urauerunt).

18. CIL, II, $2870=$ ERLara, $145:$ Marcelo / Aurelio.

19. CIL, II, $6028=C I L, \mathrm{II}^{2} / 14,590=E L S T, 271=I R S A T, 330$ : C(aius) Aemil[ius] / Niger h(ic) s(itus) [e(st)] / Rustica / Baebia / ux [or].

20. I. Kajanto (1977: 151-152).

21. Pour une liste: O. Salomies (1987: 120-131).

22. B(- - ) Camice; L. Cassius Pyramus ; P. Clodius Staphylus ; Eleuthera ; Baebia Nicostratis ; Epaphroditus ; Eutychia ; Iunia Clita. catégories sociales; c'est ainsi qu'un IIIIvir anonyme côtoie Secundilla, nourrice d'un certain Annius et très vraisemblablement une esclave.

Par ailleurs, il est impossible d'attribuer une datation précise faute d'éléments concrets dans la mesure où la présence des formules funéraires HSE et STTL, abrégées, n'autorise aucune chronologie relative. Celleci ne semble d'ailleurs en rien précoce compte tenu de la concision. Seul le recours au fragile et au contestable argument paléographique avait poussé J. González Fernández à dater l'inscription du $\mathrm{II}^{\mathrm{e}} \mathrm{s} .{ }^{23}$ puisque, comme on l'a déjà signalé, le contexte archéologique n'est ici, une nouvelle fois, d'aucun secours.

La défunte, qui s'appelait Optata Erennia, ne devait pas être apparentée aux Herennii Optati connus à Barcino (Barcelone) $)^{24}$, à Lucentum (Alicante) ${ }^{25}$ ou à Valentia (Valencia) ${ }^{26}$ en raison de l'éloignement géographique. Toutefois, elle devait se rattacher à la gens Herennia attestée à maintes reprises à Gades et dans ses environs : L. Herennius Herculaneus $(A E, 1995,817$ $=H E p, 6,464) ;$ Herennius $(A E, 1995,840=H E p, 6$, 513) ; Herennia (IRCP, 453); Herennia Crocina (CIL, II, $1821=$ IRPCadiz, $217=C L E, 1566(=I L S, 8130)$ $=$ CLECadiz, $8(=$ HEp $, 14,112=A E, 2005,715))$; $\mathrm{C}$. Herennius $(C I L$, II, $1822=I R P C a ́ d i z, 218)$; L. Herennius Anteros (CIL, II, $1823=$ IRPCadiz, 219) ; Servilius Herennius (CIL, II, $1887=I R P C a d i z, 283=H E p$, 14, 106) ; [- - He]renn[ius - - - $(H E p, 6,355)$.

Quant à la variante Erennii ${ }^{27}$, nous n'en possédons que quelques témoignages en Espagne, outre Q. Erennius Florus (parent d'Optata ?2), à Cadix, citons G. Erennius Saturninus à Augusta Emerita (Mérida) ${ }^{29}$ et L. Erennius Maurus à Carruca (Cortijo de los Cosmes) ${ }^{30}$.

23. J. González, IRPC [n. 3], p. 224.

24. $C I L$, II, 4525 (p. 982) =IRB, $54=I R C, I V, 61=A E, 1957$, 36 (Herennia C. f. Optata) ; CIL, II, $4545=I R C$, IV, 97 (C. Herennius Optatus). Un N. Herennius Optatus a vécu à Teanum Sidicinum (Italia, regio I): $C I L, \mathrm{X}, 4792$ (= ILS, $5677=A E, 1979,154)$.

25. IIlici, 89 et 90 (= $A E 1993,1065)$ (L. Herennius Optatus).

26. IValentia, 115 (L. Herennius Optatus).

27. CIL, III, 2347-2348; $10884 ; 3596 ; 2994$. CIL, V, 6662 et 6758. CIL, VI, $39450 ; 29205$ (Aerennius) $; 3743=31130=36760$; $35440 ; 11178$ (Aerennia). CIL, VIII, 19531 (Aerennius) ; 9970 (Aerennius) ; $19761 ; 20505-20506$; $9801 ; 6216$; 28053 ; 9807 (Haerennius) ; 5685 ; 25665 ; 23129 (Haerennia). CIL, IX, 6083,83 ; 2501 (3x). CIL, X, 2504-2505; 8059,149=CIL, II, 4975,30=HEp, 9,727 (de Naples bien que conservé à Madrid) ; 7556; $3484 ; 3560$; 8110 (2x); 2747. CIL, XI, 6998. CIL, XII, 5690,60. CIL, XIII, 6049 (add) ; 1401. CIL, XIV, 256,307. $A E, 1947,12 ; A E, 1963,3 ; A E$, 1967, 428; $A E, 1980,62 ; A E, 1984,213-214 ; A E, 1992,1653 ; A E$, 1997, $1686 ; A E, 2005,497$.

28. IRCP, 423

29. $H A E, 670$.

30. CILA, Se, $3,747=C I L, \mathrm{II}^{2} / 5,1148$. 
Ce gentilice, à l'instar de nombreux anthroponymes en-ennius, serait d'origine étrusque, même si les $\mathrm{He}$ rennii romains provenaient en majorité d'Arpinum ${ }^{31}$. Une autre origine est plausible puisque que Herennius correspond au gérondif osque herenno- ${ }^{32}$. Signalons aussi l'existence du gentilice Aerennius, qui n'a rien à voir avec Herennius ${ }^{33}$.

Le surnom Optata, quant à lui, est attesté dans toutes les provinces de langue latine de l'Empire ${ }^{34}$, tant en Europe ${ }^{35}$, qu'en Afrique ${ }^{36}$. En ce qui concerne plus particulièrement l'Espagne, d'après les recensements de J. M. Abascal Palazón, nous pouvons nous rendre compte que ce cognomen, outre qu'il occupe le $21^{\mathrm{e}}$ rang en fréquence avec 81 attestations, est très répandu en Bétique $^{37}$. Les recensements de S. Lefebvre confirment le premier rang de cette province, même si le nombre de témoignages avoisine les 90 cas ${ }^{38}$.

Il est également très courant parmi les esclaves et les affranchis $^{39}$, à Rome, par exemple, avec 48 exemples de “Optatus", 22 de "Optata" et 1 de "Optandus"40. En Espagne, toutes les catégories sociales sont représentées, y compris les pérégrins ${ }^{41}$.

En conclusion, la réédition de cette inscription aura permis de confirmer la lecture de son inventeur. Toutefois, des zones d'ombres, telles que l'emplacement actuel de la pièce ou encore le statut social de la défunte doivent rester en suspens, faute d'indices.

\section{BIBLIOGRAPHIE}

ABASCAL PALAZÓN, J.M. (1994): Los nombres personales en las inscripciones latinas de Hispania (Anejos de Antigüedad y Cristianismo 2). Murcia.

DENIAUX, E. (1979): "A propos des Herennii de la République et de l'époque d'Auguste", MEFRA 91 (2) : 623-650.

31. W. Schulze (1904/1966: 82-83, 282).

32. W. Schulze (1904/1966: n. 30, p. 282). C'est à une origine osque que pense aussi E. Deniaux (1979: 623).

33. W. Schulze (1904/1966: n. 30, 111-112).

34. I. Kajanto (1965: 75-76) et H. Solin (1996: 110-111).

35. B. Lörincz (2000: 114-115).

36. I. Kajanto (1965: n. 33, 76-77).

37. J.M. Abascal (1994: 444-445)

38. S. Lefebvre (2006: 123). Une nouvelle inscription d'Astigi (Écija, prov. Séville), relative à un certain Q. Avillius Pap. Optatus, renforce le premier rang détenu par la Bétique $(A E, 2006,649)$.

39. I. Kajanto (1965: 76-77, 140 cas sur 831).

40. H. Solin (1996: n. 33, 110-111).

41. S. Lefebvre (2006: n. 37, 123-124).
GONZÁLEZ FERNÁNDEZ, J. (1982): Inscripciones romanas de la provincia de Cádiz, 1982, nº 424. Cádiz.

JIMÉNEZ CISNEROS, M‥J. (1960): “Miscelánea epigráfica. Inscripciones funerarias gaditanas inéditas", Emérita 32: 295-304.

KAJANTO, I. (1965): The Latin Cognomina (Commentationes humanarum litteratum XXXVI, 2). Helsinki.

- (1977): “On the Peculiarities of Women's Nomenclature", in N. DUVAL et al. (éds.), L'onomastique latine. Paris, 13-15 octobre 1975 : 147-159. Paris.

KAJAVA, M. (1994): Roman Female Praenomina. Studies in the Nomenclature of Roman Women (Acta instituti Romani Finlandiae 14). Rome.

LEFEBVRE, S. (2006): "Les migrations des Africani en péninsule ibérique: quelle vérité ?", in A. Caballos Rufino et S. Demougin (éds.), Migrare. La formation des élites dans l'Hispanie romaine. Bordeaux.

LÓPEZ DE LA ORDEN, Ma.D. (2001): De epigraphia Gaditana. Cadix.

LÓPEZ DE LA ORDEN, Mª.D. et RUIZ CASTELLANOS, A. (1995): Nuevas inscripciones latinas del Museo de Cádiz. Cádiz.

LÖRINCZ, B. (2000): Onomasticon prouinciarum Europae latinarum III. Budapest-Vienne.

NAVARRO CABALLERO, M. et al. (2003): Atlas antroponímico de la Lusitania romana. MéridaBordeaux.

PARODI ÁlVAREZ, M.J. (2002): Puerto Real en la Historia. Ensayo de Bibliografía Histórica Portorrealeña. Sevilla.

- (2006): Puerto Real en la Historia (II). Nuevo Ensayo de Bibliografía Histórica Portorrealeña. Puerto Real.

SALOMIES, O. (1987): Die römischen Vornamen. Studien zur römischen Namengebung. Helsinki (Commentationes humanarum litterarum 82).

SCHULZE, W. (1904/1966): Zur Geschichte lateinischer Eigennamen. Göttingen (1904 éd. or.). BerlinZürich-Dublin (rééd. 1966).

SOLIN, H. (1996): Die stadtrömischen Sklavennamen. Ein Namenbuch I-III. (Forschungen zur antiken Sklaverei. Beiheft 2). Mayence.

VALLEJO RUIZ, J.M. (2005): Antroponimia indigena de la Lusitania romana (Anejos de Veleia, Serie minor 23). Vitoria.

Fecha DE ENTRADA: 1-02-2011

FECHA DE ACEPTACIÓN: 23-03-2011 